\title{
Association Between Body Mass Index and Male Sperm Apoptosis and Apoptosis-Related Factors
}

This article was published in the following Dove Press journal:

Diabetes, Metabolic Syndrome and Obesity: Targets and Therapy

\section{Guangli Zhu \\ Yuting Zhang \\ Jianjun Dong \\ Yilin Liu \\ Fang Zhao \\ Ting Li \\ Zhanrong Shi \\ Yanping Zhang \\ $\mathrm{Na}$ Song \\ Wenyue Song \\ Yuzhen Lv}

Reproductive Medicine Center, Jiaozuo Women and Children's Hospital, Jiaozuo, Henan Province, People's Republic of China
Correspondence: Yuzhen Lv Reproductive Medicine Center, Jiaozuo Women and Children's Hospital, Jiaozuo, 454I50, Henan, People's Republic of China

Tel +86I3839|58938

Email lyz jiaozuo@।26.com
Background: Although obesity may affect reproductive functions, the molecular mechanisms of apoptosis-related biomarkers remain uncertain.

Objective: To examine the effects of body mass index on sperm quality and apoptosisrelated factors in seminal plasma of men.

Methods: Data for 54 subfertile men were collected at our reproductive medical center. The men were divided into normal weight, overweight, and obese groups based on their body mass index (BMI). Sperm DNA fragmentation (sperm chromatin structure analysis), sperm apoptosis (annexin V), and sperm apoptosis-related factors (antibody array assay) were assessed and their relationships with BMI were analyzed.

Results: BMI was not significantly related to age, duration of infertility, duration of sexual abstinence, semen volume, sperm concentration, or rate of normal sperm morphology ( $p>$ 0.05 ). However, progressive sperm motility was significantly reduced and the rates of sperm DNA fragmentation index (DFI) and sperm apoptosis were significantly increased in overweight and obese men compared with men with normal BMI. Fas/Fasl, Bcl-2/Bax, caspase-3, caspase-8, p53, and p21 were all upregulated in the overweight and obese groups. Protein function annotation by Gene Ontology analysis and Kyoto Encyclopedia of Genes and Genomes pathway analysis revealed that apoptosis-related factors were enriched in a network associated with activation of apoptotic signaling pathways, such as apoptosis and p53 signaling.

Conclusion: These data suggest that increased BMI is associated with increased sperm apoptosis and sperm DNA damage, as well as accelerated expression of apoptosis-related factors via the activation of apoptotic signaling pathways.

Keywords: BMI, obesity, semen quality, sperm DNA, apoptosis-related factors, biomarker

\section{Introduction}

Overweight and obesity have become major public health concerns worldwide as a result of changes in current lifestyles, with alarming increases in the numbers of overweight and obese individuals in developed countries. ${ }^{1,2}$ Increased body weight has been associated with multiple inter-related disorders, including hypertension, cardiovascular disease, type 2 diabetes, and other metabolic syndromes. ${ }^{3,4}$ Obesity may also affect reproductive functions. For example, previous studies reported an association between body weight and various standard semen-analysis parameters, although these findings have been inconsistent. ${ }^{5,6}$ There is limited information to date on the effect of body weight on the integrity of sperm DNA and apoptosis. ${ }^{7}$

Levels of inflammatory cytokines in seminal plasma are known to be altered in overweight and obese men. Specifically, obesity has been associated with elevated 
levels of inflammatory adipocytokines, such as interleukin 6 (IL-6) and tumor necrosis factor alpha (TNF- $\alpha$ ), which can cause sperm cell apoptosis. However, the exact role of altered apoptosis-related protein expression in male reproductive potential remains unknown. In addition, spermatogenesis may be affected by upstream and/or downstream changes in seminal plasma in obese males. Based on these facts, we aimed to explore the relationships between sperm apoptosis and sperm DNA damage and apoptotic signaling pathways in obese men.

\section{Methods}

\section{Study Population}

Males ranging in age from 22 to 40 years who presented for evaluation at the Reproductive Center of Jiaozuo Women and Children's Hospital over the period between July and December of 2019 were matched as based on their subfertile status. Three groups of males (normal weight, overweight and obese) based upon BMI levels were compared in this study. ${ }^{8,9}$ All participants were asked to provide detailed information on occupation, medical and reproductive history and lifestyle. Inclusion criteria is the age from 22 to 40 years, the $B M I \geq 18.5$ and they had normal sexual life without contraception and had not given birth for at least 1 year. Exclusion criteria included regular alcohol drinkers, heavy smokers, chronic diseases, azoospermia and any other diseases which might lead to dysspermia. The study was approved by the Institutional Ethical Committee of Jiaozuo Women and Children's Hospital. The study was in compliance with the Declaration of Helsinki for clinical research. All participants provided written informed consent before participating in the study.

\section{Sample Collection}

Semen specimens were collected by masturbation after a 2-7 day period of sexual abstinence and were maintained to liquefy at $37^{\circ} \mathrm{C}$ for $30 \mathrm{~min}$. After liquefaction, semen volume was measured by weighing the sample, while sperm concentration, total motility and progressive sperm motility were analyzed using a computer-aided sperm analysis (CASA) system (WLJY-9000; Sperm color analysis system, Beijing, China). ${ }^{10}$

\section{Determination of Morphology}

A minimum of 200 motile spermatozoa per sample were obtained for evaluation and determination of percent of normal spermatozoa.

\section{Determination of Sperm DNA}

\section{Fragmentation}

Sperm chromatin structure analysis (SCSA) and flow cytometry were used to detect the sperm DNA fragmentation (SDF). After acid treatment of semen, the resultant structural damage would enable the double-stranded sperm DNA to unwind. BD flow cytometry was then used to detect the fluorescent signal, with the degree of red fluorescence providing an index of the degree of sperm damage. The DNA fragmentation index (DFI) value was then calculated with use of CASA-WLJW-9000 according to the following formula: DFI value of single sperm $=\mathrm{red} /$ (red + green) $* 100 \%$. This value represented the ratio of denatured sperm DNA/total DNA. ${ }^{11}$

\section{Determination of Sperm Apoptosis}

Sperm suspensions $\left(1 \times 10^{6}\right.$ cells $\left./ \mathrm{mL}\right)$ were incubated at room temperature for $15 \mathrm{~min}$ in the dark within an appropriate binding buffer containing $1 \mu \mathrm{L}$ annexin $\mathrm{V}$ (green), 1 $\mu \mathrm{L}$ propidium iodide (PI) (red) and $1 \mu \mathrm{L}$ of cell-permeable DNA stain Hoechst 33,342 (blue). The PI dye was impermeable to live cells. With this procedure, subpopulations of spermatozoa could be identified such that, annexin $\mathrm{V}(-) / \mathrm{PI}(-)$ indicated live intact sperm, annexin $\mathrm{V}(+) / \mathrm{PI}(-)$ - early apoptotic cells and annexin $\mathrm{V}(+/-) / \mathrm{PI}(+)$ - necrotic cells. The percent of early apoptotic cells were determined. At least 200 spermatozoa were evaluated within each slide, as achieved with use of an appropriate filter.

\section{Antibody Array Assay}

With this assay it was possible to simultaneously detect 43 separate cytokines. According to the internal positive controls provided by the RayBiotech analysis tool, signal values were read and normalized.

\section{Statistical Analysis}

All data analyses were performed using SPSS 21.0 software (SPSS Inc., Chicago, IL, USA). Data are presented as mean \pm standard deviation (SD). A one-way ANOVA followed by the Newman-Keuls post hoc test were used to analyze differences among the groups. A P-value $<0.05$ was required for results to be considered statistically significant.

\section{Results}

Table 1 contains a summary of the characteristics and semen quality parameters of the 54 men recruited for this study. The data from these participants were allocated into 
Table I Characteristics of the Study Population by Body Mass Index ( $N=54)$

\begin{tabular}{|c|c|c|c|c|}
\hline Group (N) & $|8.5 \leq \mathrm{BM}|<24(16)$ & $24 \leq B M \mid<28$ (I 7$)$ & $B M I \geq 28$ (2I) & $P$ value \\
\hline Age (yr) & $29.38 \pm 3.57$ & $30.29 \pm 5.43$ & $32.38 \pm 5.26$ & 0.164 \\
\hline Infertility time (yr) & $2.69 \pm 1.08$ & $2.06 \pm 0.90$ & $3.52 \pm 3.14$ & 0.111 \\
\hline Abstinence time (days) & $4.44 \pm 1.03$ & $4.88 \pm 1.93$ & $5.00 \pm 1.79$ & 0.576 \\
\hline Semen vol (mL) & $3.06 \pm 1.19$ & $2.49 \pm 1.03$ & $3.06 \pm 0.79$ & 0.156 \\
\hline Sperm conc $(\times 106 / \mathrm{mL})$ & $36.50 \pm 19.62$ & $42.17 \pm 35.65$ & $52.08 \pm 36.32$ & 0.332 \\
\hline Progressive motility (\%) & $48.92 \pm|5.7|$ & $42.36 \pm 16.00$ & $32.75 \pm 14.80^{*}$ & 0.009 \\
\hline Normal morphology (\%) & $2.06 \pm 1.69$ & $2.65 \pm 2.13$ & $3.00 \pm 2.07$ & 0.372 \\
\hline Sperm DFI (\%) & $12.49 \pm 7.28$ & $12.89 \pm 9.48 * *$ & $30.97 \pm 18.10 *$ & $<0.001$ \\
\hline Sperm apoptosis (\%) & $2.98 \pm 2.01$ & $6.02 \pm 4.82 * *$ & $9.35 \pm 5.72^{*}$ & 0.001 \\
\hline
\end{tabular}

Notes: *Significant difference was found compared with $\mid 8.5 \leq \mathrm{BMI}<24$. **Significant difference was found compared with $\mathrm{BMI} \geq 28$.

one of the three groups as based upon their BMI levels as described above. Our results revealed that BMI did not show any significant relationship as related to age of the participants, infertility time, time of sexual abstinence, sperm volume, sperm concentration and rate of normal morphology $(p>0.05)$. However, sperm progressive motility, sperm DFI and sperm apoptosis were all found to be altered as a function of increased BMI values $(p<0.05)$.

Representative images of sperm DFI and sperm apoptosis are shown in Figures 1 and 2, respectively.

Expression Characteristics of apoptosis-related factors in seminal plasma of men, Figure 3A contains a map of the apoptosis-related antibody array $\mathrm{G}$ series I including the 43 cytokines detected. Figure 3B, the array distribution of positive correlation between fluorescent intensities and expression levels, further revealing the variations of these proteins as observed in the three BMI groups.

\section{Altered Apoptosis-Related Factor Levels in the Overweight versus Normal Weight Group}

The parameter used for evaluating statistical significance involved that of determining the fold change. For this analysis, results included $(\log 2)$ fold changes for each protein and for each individual contrast. Differentially expressed proteins (DEPs) were defined as those demonstrating a fold change of $<0.83$ or $>1.2$ (absolute $\log \mathrm{FC}>0.263$ ). Based on this analysis, we found that cytoC, IGFBP-4, Fas, IGFBP-3, DR6, bcl-2, IGFBP-2, TRAILR-2, HTRA, bad, caspase-8, CD40L, Caspase-3, SMAC, BID, FasL, BIM, bcl-w, CD40, TRAILR-1, XIAP, Survivin, HSP60, IGFBP-6, cIAP2, IGFBP-1, IGFBP-5, IGF-I, IGF-II, sTNF-R1, bax, HSP27, p27, p53, TNF- $\beta$, HSP70, IGF-1sR, livin,
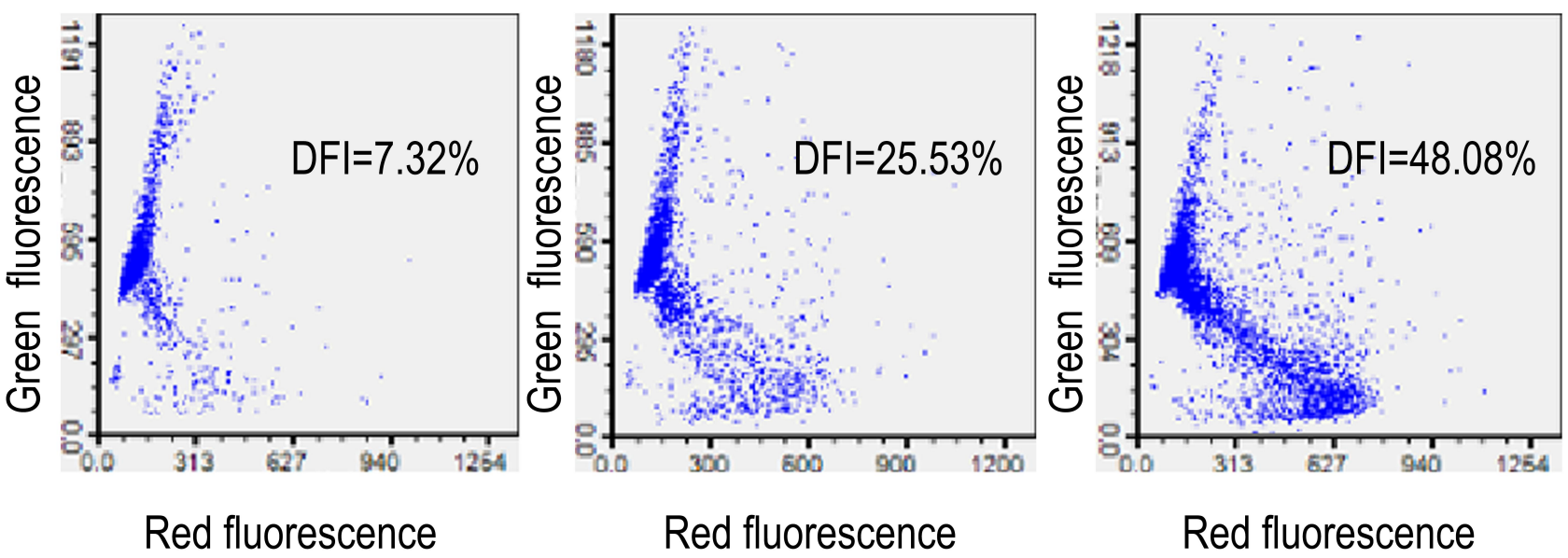

Figure I Sperm DFI as determined using flow cytometry and assessed with use of a sperm chromatin structure assay (SCSA). Three different levels of sperm DNA fragmentation rate are shown. 


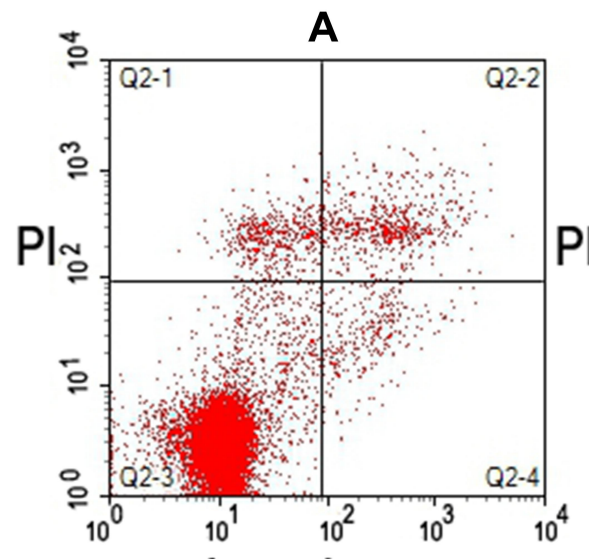

Annexin-v

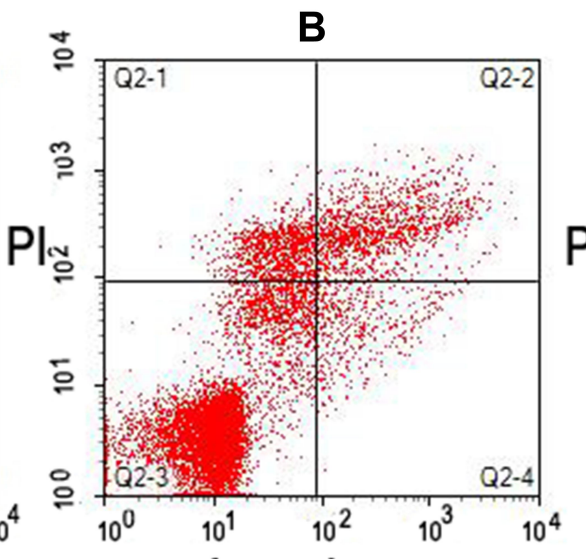

Annexin-v

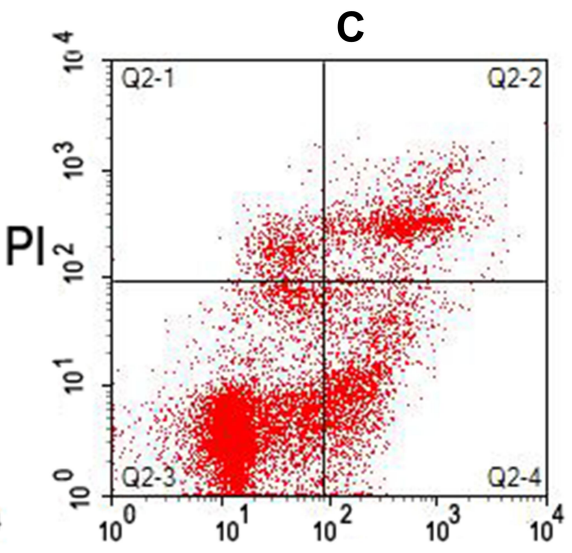

Annexin-v

Figure 2 Sperm apoptosis as determined using flow cytometry. (A and B) Lower levels of apoptosis ( $2.82 \%$ and $3.02 \%$, respectively). (C) Higher levels of apoptosis $(17.46 \%)$.

\section{A}

\begin{tabular}{|c|c|c|c|c|c|c|c|c|c|c|c|c|c|}
\hline Pos & Pos & Neg & Neg & Blank & Blank & bad & bax & bcl-2 & bcl-w & BID & BIM & Caspase3 & caspase8 \\
\hline Pos & Pos & Neg & Neg & Blank & Blank & bad & bax & bcl-2 & bcl-w & BID & BIM & Caspase3 & caspase8 \\
\hline CD40 & CD40L & cIAP-2 & cytoC & DR6 & Fas & FasL & Blank & HSP27 & HSP60 & HSP70 & HTRA & IGF-I & IGF-II \\
\hline CD40 & CD40L & cIAP-2 & cytoC & DR6 & Fas & FasL & Blank & HSP27 & HSP60 & HSP70 & HTRA & IGF-I & IGF-II \\
\hline IGFBP-1 & IGFBP-2 & IGFBP-3 & IGFBP-4 & IGFBP-5 & IGFBP-6 & IGF-lsR & livin & p21 & p27 & p53 & SMAC & Survivin & sTNF-R1 \\
\hline IGFBP-1 & IGFBP-2 & IGFBP-3 & IGFBP-4 & IGFBP-5 & IGFBP-6 & IGF-lsR & livin & p21 & p27 & p53 & SMAC & Survivin & sTNF-R1 \\
\hline sTNF-R2 & TNF-alpha & TNF-beta & TRAIIR-1 & TRAILR-2 & TRAILR-3 & TRAIIR-4 & XIAP & Blank & Blank & Neg & Neg & Neg & Pos \\
\hline sTNF-R2 & TNF-alpha & TNF-beta & TRAIIR-1 & TRAIIR-2 & TRAILR-3 & TRAIIR-4 & XIAP & Blank & Blank & Neg & Neg & Neg & Pos \\
\hline
\end{tabular}

\section{B Normal weight group}

\section{Overweight group Obesity group}

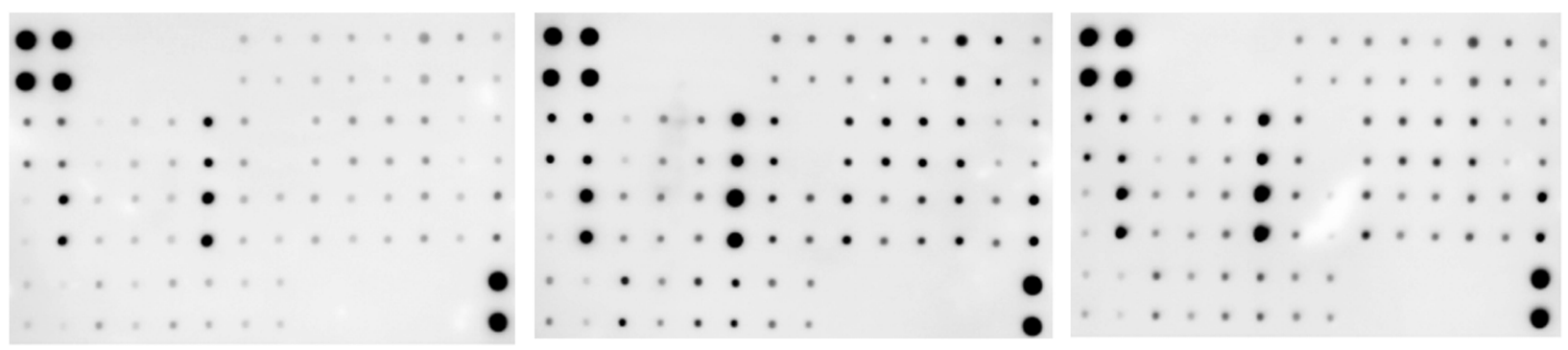

Figure 3 Antibody array profiles and expression levels of apoptosis-related factors. (A) A Map of mouse inflammation antibody array G series I including 43 cytokines. (B) The levels of cytokines are proportional to their fluorescent intensities.

sTNF-R2, TNF- $\alpha$, TRAILR-4, TRAILR-3 and p21 were all significantly upregulated in the overweight group as compared with that in normal weight group
(Figure 4A and $\mathrm{B}, \mathrm{P}<0.05$ ). In contrast, none of these proteins was significantly downregulated in the overweight group $(\mathrm{P}>0.05)$. 
A

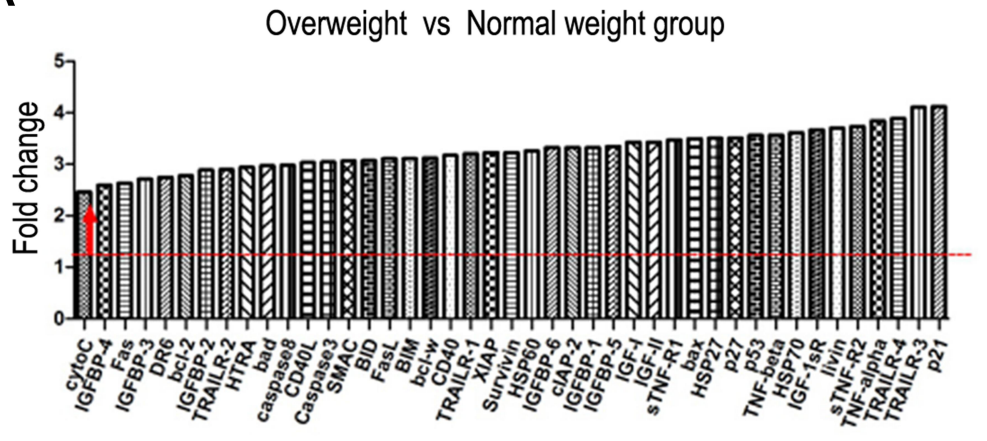

B

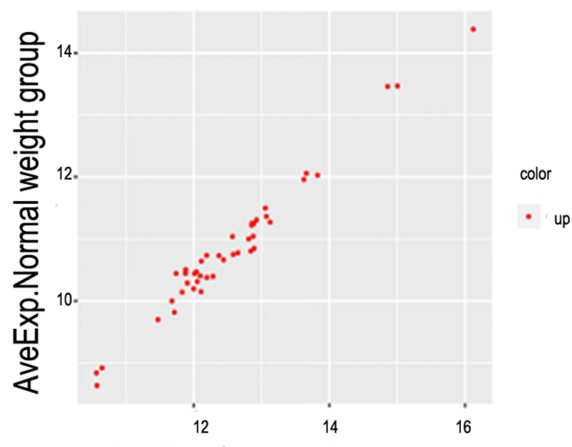

AveExp.Overweight group

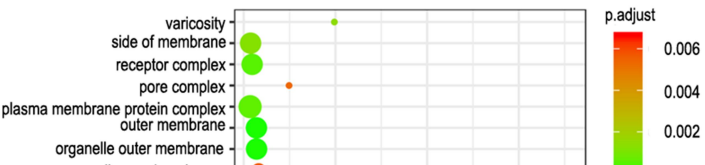

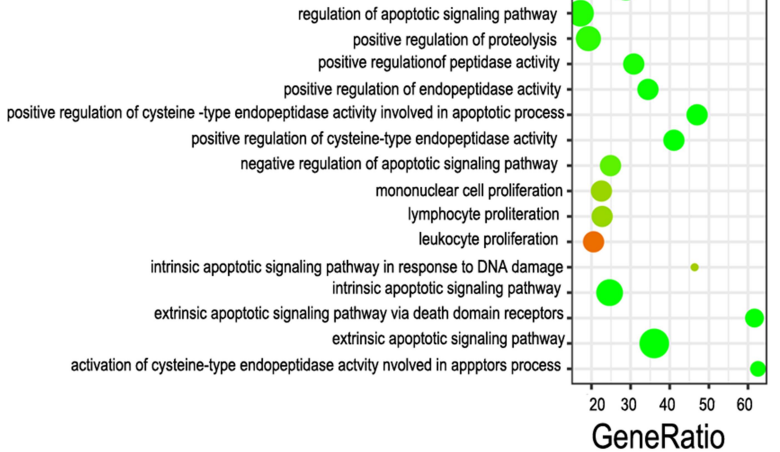

$$
\text { c }
$$
regulation of endopeptidase activity regulation of cysteine-type endopeptidase activity involved in apoptotic process
regulation of cysteine-type endopeptidase activity $\begin{array}{r}\text { regulation of apoptotic signaling pathway } \\ \text { positive regulation of proteolysis } \\ \text { positive regulationof peptidase activity } \\ \text { positive regulation of endopetidase activity } \\ \text { positive regulation of cysteine -type endopeptidase activity involved in apoptotic process. } \\ \text { positive regulation of cysteine-type endopeptidase activity } \\ \text { negative regulation of apoptotic signaling pathway } \\ \text { mononuclear cell proliferation } \\ \text { lymphocyte proliteration } \\ \text { leukocyte proliferation }\end{array}$
intrinsic apoptotic signaling pathway in response to DNA damage
intrinsic apoptotic signaling pathway
extrinsic apoptotic signaling pathway via death domain receptors.
extrinsic apoptotic signaling pathway

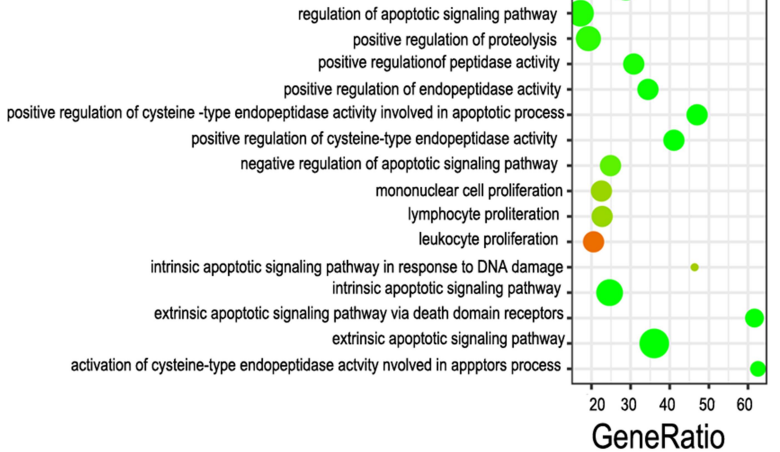

E

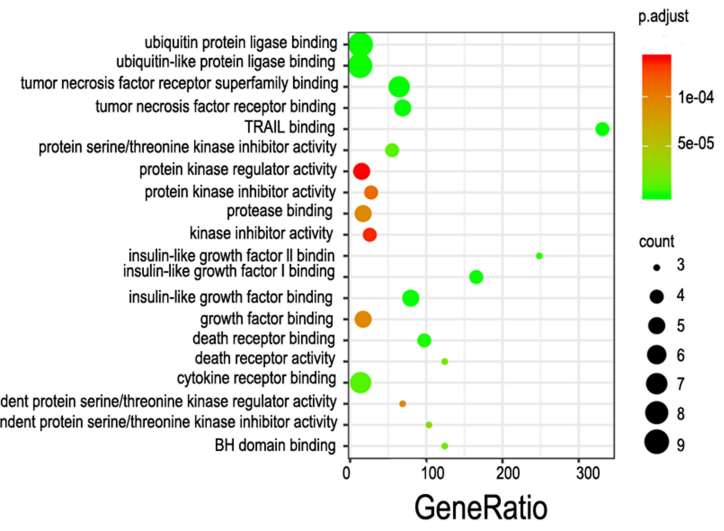

p.adjust $\quad$ D $3 \mathrm{e}-12$ $2 \mathrm{e}-12$ $1 \mathrm{e}-12$

membrane microdomain

r binding poten complex growth factor complex death-inducing signaling complex CD95 death-inducing signaling complex
CD40 receptor complex

Bcl-2 family protein complex

$$
0
$$

100

$\mathbf{F}_{\text {Vira }}$

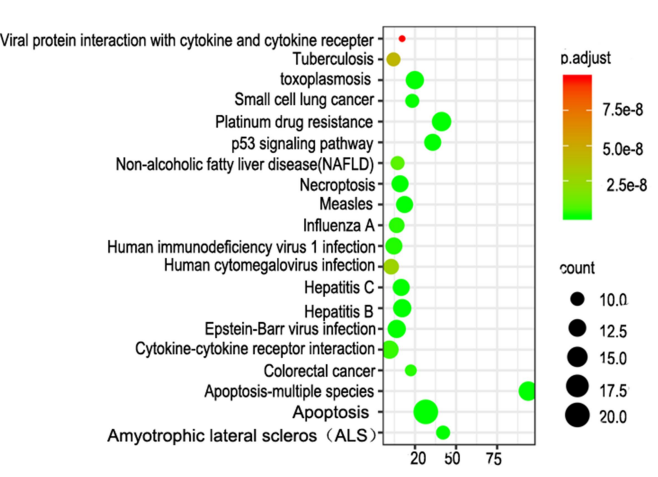

Figure 4 Protein function annotation GO and KEGG pathway analysis in the overweight versus normal weight group. (A) DEPs. (B) Scatter plot of apoptosis-related factors with red indicating upregulation, blue downregulation and gray no difference. (C) Biological process (D) Cellular component and (E) Molecular function. (F) KEGG analysis of protein functions, linking genomic information with higher-order functional information.

In order to better understand the role of apoptosisrelated factors in seminal plasma as related to body mass index, protein function annotation gene ontology $(\mathrm{GO})$ and the KEGG pathway were analyzed with the use of the R package "clusterProfiler." GO analysis included three subtypes: BP (biological process, Figure 4C), MF (molecular function, Figure 4E) and CC (cellular component, Figure 4D). KEGG involved a systematic analysis of protein functions, linking genomic information with higher-order functional information (Figure 4F). When comparing apoptosis-related factors between the overweight and normal weight group, we found that those of the overweight group were enriched in a network associated with activation of apoptosis-related pathways such as cytokine-cytokine receptor interaction and the p53 signaling pathway. 


\section{Altered Apoptosis-Related Factor Levels in the Obesity versus Normal Weight Group}

As shown in Figure 5A, livin was significantly downregulated in the obesity as compared with the normal weight group (Figure 5B, $\mathrm{P}<0.05$ ). In contrast, CD40, TRAILR-2, CD40L, SMAC, bad, sTNF-R2, IGFBP-3, IGFBP-4, Fas, Caspase-3, IGFBP-2, TNF- $\beta$, FasL, BID, DR6, HSP60, cytoC, BIM, bclw, HTRA, bcl-2, p53, TRAILR-1, XIAP, HSP70, HSP27, bax, p27, caspase-8, cIAP-2, TRAILR-3, TNF- $\alpha$, IGF-1sR, IGF-II,
IGF-I, IGFBP-6, TRAILR-4, Survivin, sTNF-R1, IGFBP-5, IGFBP-1 and p21 were all significantly upregulated in the obesity group $(\mathrm{P}<0.05)$. Protein function annotation $\mathrm{GO}$ (Figure 5C-E) and KEGG pathway (Figure 5F) results showed that apoptosis-related factors within both the obesity and normal weight groups were enriched in a network associated with activation of apoptosis-related pathways, for example, cytokine-cytokine receptor interactions, the p53 signaling pathway, necroptosis and apoptosis.
A
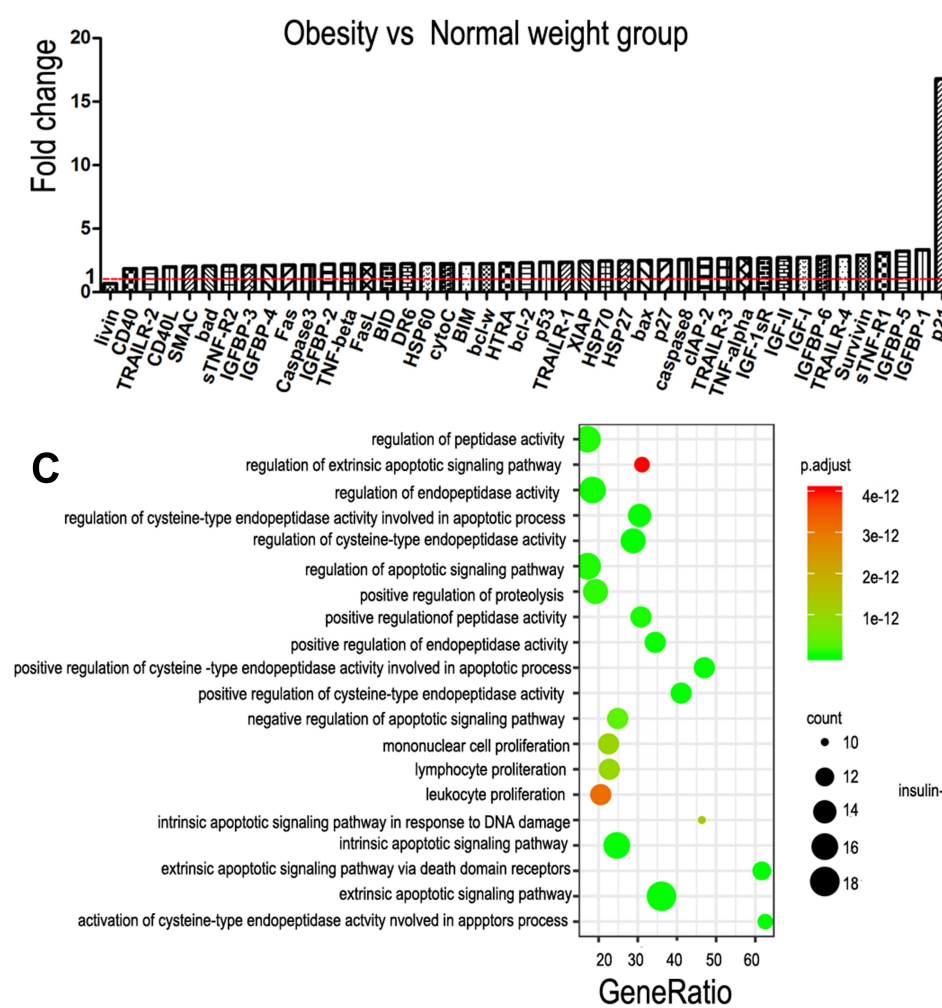

E
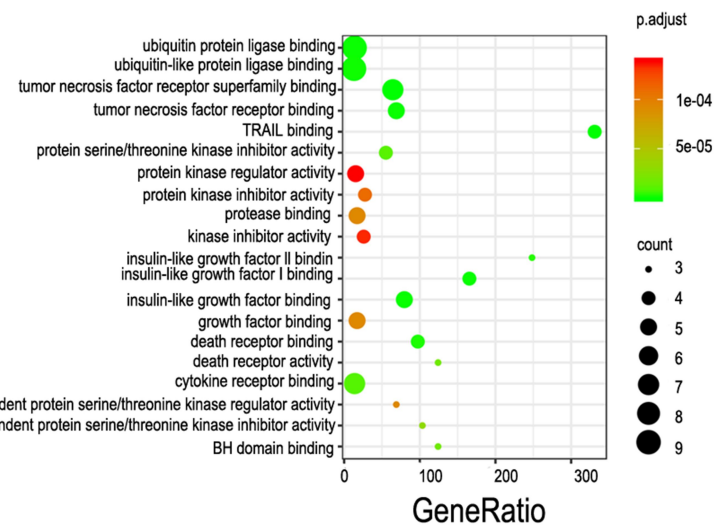

$\mathbf{F}$
B

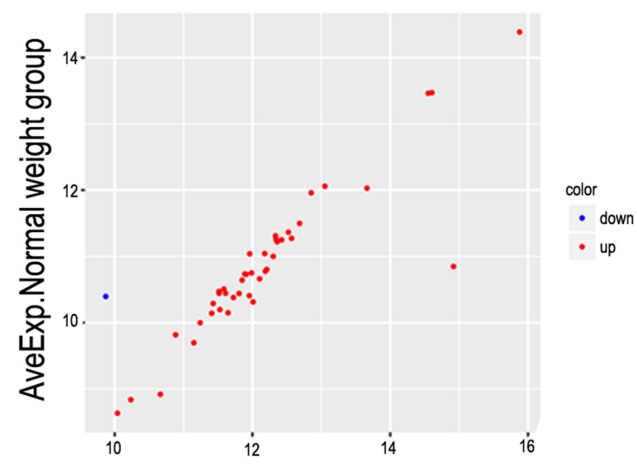

D

AveExp.Overweight group

e-12

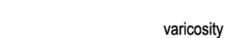

varicosity
side of membrane
receptor complex

pore complex
plasma membrane protein complex outer membrane $1 e-12$

count 10

12 insulin-like growth membrane microdomain sulin-like growth factor binding poten complex
growth factor complex external side of plasma membrane death-inducing signaling complex CD95 death-inducing signaling complex
CD40 receptor complex Bcl-2 family protein complex $\begin{array}{lll} & 100 & 200 \\ & \text { GeneRatio }\end{array}$

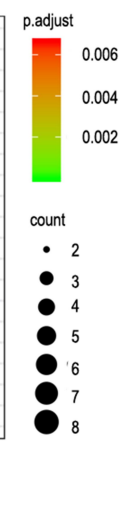

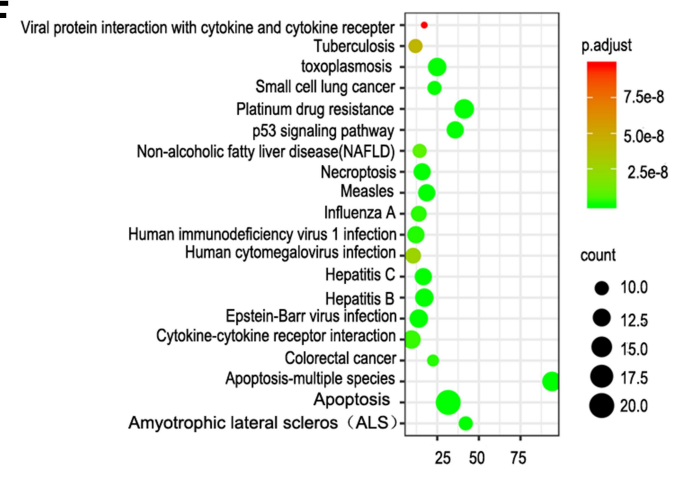

Figure 5 Protein function annotation GO and KEGG pathway analysis in the obesity versus normal weight group. (A) DEPs. (B) Scatter plot of apoptosis-related factors with red indicating upregulation, blue downregulation and gray no difference. (C) Biological process. (D) Cellular component and (E) Molecular function. (F) KEGG analysis of protein functions, linking genomic information with higher-order functional information. 


\section{Discussion}

This study evaluated the associations between BMI and multiple parameters of male semen quality in a fertility clinic in China. The results suggested that progressive sperm motility was decreased in obese men compared with men with a normal BMI, with no significant difference between overweight and obese men. This was in accord with the results of other studies that found a significant reduction in total and/or progressive sperm motility in parallel with increases in BMI., ${ }^{5,12}$ Furthermore, the percent of sperm DNA fragmentation index and sperm apoptosis (early apoptotic spermatozoa) were both significantly increased in overweight and obese men, consistent with previous studies reporting increased sperm DNA fragmentation in obese patients. ${ }^{5,13,14}$ In contrast, some previous studies found that obesity had little or no influence on either sperm apoptosis or sperm DNA integrity. ${ }^{15,16,19}$ On the other hand however, the current results found no significant relationship between BMI and sperm volume, sperm concentration, or rate of normal sperm morphology. This was in agreement with some previous studies, which failed to demonstrate any significant effect of BMI on semen volume, ${ }^{17,18}$ or on sperm concentration or morphology. ${ }^{13,19-21}$

Obesity has been closely related to male subfertility; ${ }^{22}$ however, the molecular basis for this negative influence of obesity on semen quality remains unclear. ${ }^{23,24}$ In this study, we used a relatively novel approach involving antibody microarray technology. This technique can be applied for the simultaneous detection of multiple proteins, and has the advantage of high-throughput applicability. ${ }^{25} \mathrm{We}$ accordingly used this advanced antibody array detection chip technology to identify protein markers associated with sperm apoptosis and sperm damage, and showed that Fas/Fasl, Bcl-2/Bax, caspase-3, caspase- 8 , p53, p21, TNF- $\alpha$, TNF- $\beta$, sTNF-R1, and sTNFR2 were all significantly upregulated in line with increasing BMI in men. Interestingly, livin, which was upregulated in the overweight group, was significantly downregulated in the obese group. These results reveal some of the apoptosisrelated factors that are activated in response to sperm apoptosis and sperm damage.

Previous clinical and animal studies have shown that obesity is correlated with male subfertility. ${ }^{26,27}$ Adipokines such as TNF- $\alpha$ and IL-1, IL-6, and IL-18, which are produced by adipose tissue, have been reported to induce severe inflammation. ${ }^{28}$ The current results showed that sTNF-R2, TNF- $\beta$, and TNF- $\alpha$ were all significantly upregulated with increasing BMI. These findings were similar to those of previous studies that showed increased TNF- $\alpha$ and IL- 6 levels in serum, testicular tissue, and seminal plasma in obese men. ${ }^{29,30}$ Obesity is associated with a chronic inflammatory state and is accompanied by increased formation of reactive oxygen species (ROS) in testicular tissue, the reproductive tract, and semen. ROS represent normal products involved in cellular metabolism; however, excessive levels can induce oxidative stress resulting in DNA damage in sperm and increasing stress within the testicular environment. ${ }^{31}$

To clarify the role of these apoptosis-related factors in sperm apoptosis and sperm damage, we carried out GO protein function annotation and KEGG pathway analyses using the $\mathrm{R}$ package. The results revealed that apoptosisrelated factors within the overweight and obese groups were enriched in a network associated with the activation of apoptosis-related signaling pathways, such as apoptosis and the $\mathrm{p} 53$ signaling pathway.

Apoptosis is an autonomous programmed cell death process that is stimulated under specific conditions and which is regulated by various genes. Apoptosis of testicular spermatogenic cells represents one of the main causes of male subfertility. ${ }^{32}$ The obese group in the current study showed increased rates of sperm DNA fragmentation and sperm apoptosis, while apoptosis-related proteins, notably Fas/ Fasl, Bcl-2/Bax, caspase-3, and caspase-8, were also significantly upregulated in obese and overweight men. Another recent study found that a high-fat diet, which can result in apoptosis, was associated with increases in Bax and caspase3 expression and reductions in Bcl-2 expression within the testis, resulting in apoptosis. ${ }^{33}$ The apoptotic pathway can also be initiated by caspase- 8 , culminating in the activation of caspases- $3,-6$, and $-7 . .^{34,35}$ Interestingly, livin, as an apoptosis inhibitor, was significantly upregulated in the overweight group but significantly downregulated in the obese group. This downregulation of livin in obese men may contribute to activation of the apoptotic pathway.

The p53 signaling pathway is inactivated in most human tumors, demonstrating its critical function as a tumor suppressor gene. This pathway is normally activated by cellular stress and mediates a growth-suppressive response involving cell cycle arrest and apoptosis. In the current study, apoptosis-related proteins, such as p27, p53, and $\mathrm{p} 21$, were all significantly upregulated in overweight and obese men. These findings indicated that DNA damage in adipocytes in obese individuals could trigger p53-dependent signals involved in the alteration of adipocyte metabolism and secretory function, potentially contributing to adipose tissue inflammation and eventual induction of apoptosis. ${ }^{36}$ 
In conclusion, the results of this study indicate that progressive sperm motility is decreased in obese men, while sperm DFI and sperm apoptosis (early apoptotic spermatozoa) were increased in overweight and obese men relative to that in normal-weight men. Moreover, Fas/Fasl, Bcl-2/Bax, livin and p53 may all serve as significant biomarkers in response to sperm apoptosis and sperm damage. Finally, activation of the signaling pathways of apoptosis and p53 likely play key roles in sperm apoptosis and sperm damage within obese men.

\section{Funding}

This work was supported by the joint construction fund of Henan Provincial Medical Science and Technology Tackling Program of China (LHGJ20191362). The funding body was not involved in the design of the study, the collection, analysis, and interpretation of data and the preparation of the manuscript.

\section{Disclosure}

The authors declare that they have no conflict of interests.

\section{References}

1. Yanovski JA. Obesity: trends in underweight and obesity-scale of the problem. Nat Rev Endocrinol. 2018;14(1):5-6. doi:10.1038/ nrendo.2017.157

2. Mintziori G, Nigdelis MP, Mathew H, et al. The effect of excess body fat on female and male reproduction. Metabolism. 2020;107:154193. doi:10.1016/j.metabol.2020.154193

3. Flegal KM, Kit BK, Orpana H, Graubard BI. Association of all-cause mortality with overweight and obesity using standard body mass index categories: a systematic review and meta-analysis. JAMA. 2013;309 (1):71-82. doi:10.1001/jama.2012.113905

4. Bhaskaran K, Dos-Santos-Silva I, Leon DA, et al. Association of BMI with overall and cause-specific mortality: a population-based cohort study of 3.6 million adults in the UK. Lancet Diabetes Endocrinol. 2018;6(12):944-953. doi:10.1016/S2213-8587(18)30288-2

5. Taha EA, Sayed SK, Gaber HD, et al. Does being overweight affect seminal variables in fertile men? Reprod Biomed Online. 2016;33 (6):703-708. doi:10.1016/j.rbmo.2016.08.023

6. Umul M, Kose SA, Bilen E, et al. Effect of increasing paternal body mass index on pregnancy and live birth rates in couples undergoing intracytoplasmic sperm injection. Andrologia. 2015;47(3):360-364. doi:10.1111/and.12272

7. Kort HI, Massey JB, Elsner CW, et al. Impact of body mass index values on sperm quantity and quality. $J$ Androl. 2006;27(3):450-452. doi: $10.2164 /$ jandrol.05124

8. Zhou BF. Cooperative meta-analysis group of the working group on obesity in China. Predictive values of body mass index and waist circumference for risk factors of certain related diseases in Chinese adults-study on optimal cut-off points of body mass index and waist circumference in Chinese adults. Biomed Environ Sci. 2002;15(1):83-96.

9. Chen C, Lu FC. Department of disease control ministry of health, PR China. The guidelines for prevention and control of overweight and obesity in Chinese adults. Biomed Environ Sci. 2004;17:1-36.
10. Lu JC, Huang YF, Lu NQ. Computer-aided sperm analysis: past, present and future. Andrologia. 2014;46(4):329-338. doi:10.1111/ and. 12093

11. Jin J, Pan C, Fei Q, et al. Effect of sperm DNA fragmentation on the clinical outcomes for in vitro fertilization and intracytoplasmic sperm injection in women with different ovarian reserves. Fertil Steril. 2015;103(4):910-916. doi:10.1016/j.fertnstert.2015.01.014

12. Tang WH, Zhuang XJ, Ma LL, et al. Correlation between body mass index and semen quality in male infertility patients. Turk $J$ Med Sci. 2015;45(6):1300-1305. doi:10.3906/sag-1408-7

13. Dupont C, Faure C, Sermondade N, et al. Obesity leads to higher risk of sperm DNA damage in infertile patients. Asian J Androl. 2013;15 (5):622-625. doi:10.1038/aja.2013.65

14. Fariello RM, Pariz JR, Spaine DM, et al. Association between obesity and alteration of sperm DNA integrity and mitochondrial activity. $B J U$ Int. 2012;110(6):863-867. doi:10.1111/j.1464-410X.2011.10813.x

15. Oliveira JBA, Petersen CG, Mauri AL, et al. Association between body mass index and sperm quality and sperm DNA integrity. A large population study. Andrologia. 2018;50(3):e12889. doi:10.1111/ and.12889

16. Leisegang K, Henkel R. The impact of male overweight on semen quality and outcome of assisted reproduction. Asian $J$ Androl. 2014;16(5):787. doi:10.4103/1008-682X.133321

17. Alshahrani S, Ahmed AF, Gabr AH, et al. The impact of body mass index on semen parameters in infertile men. Andrologia. 2016;48 (10):1125-1129. doi:10.1111/and.12549

18. Campbell JM, Lane M, Owens JA, Bakos HW. Paternal obesity negatively affects male fertility and assisted reproduction outcomes: a systematic review and meta-analysis. Reprod Biomed Online. 2015;31(5):593-604. doi:10.1016/j.rbmo.2015.07.012

19. Bandel I, Bungum M, Richtoff J, et al. No association between body mass index and sperm DNA integrity. Hum Reprod. 2015;30 (7):1704-1713. doi:10.1093/humrep/dev111

20. Hajshafiha M, Ghareaghaji R, Salemi S, Sadegh-Asadi N, SadeghiBazargani H. Association of body mass index with some fertility markers among male partners of infertile couples. Int J Gen Med. 2013;6:447-451. doi:10.2147/IJGM.S41341

21. Chavarro JE, Toth TL, Wright DL, et al. Body mass index in relation to semen quality, sperm DNA integrity, and serum reproductive hormone levels among men attending an infertility clinic. Fertil Steril. 2010;93(7):2222-2231. doi:10.1016/j.fertnstert.2009.01.100

22. Cabler S, Agarwal A, Flint M, Du Plessis SS. Obesity: modern man's fertility nemesis. Asian J Androl. 2010;12(4):480-489. doi:10.1038/ aja. 2010.38

23. Nowicka-Bauer K, Kurpisz M. Current knowledge of the human sperm proteome. Expert Rev Proteomics. 2013;10(6):591-605. doi:10.1586/14789450.2013.846221

24. Amaral A, Castillo J, Ramalho-Santos J, Oliva R. The combined human sperm proteome: cellular pathways and implications for basic and clinical science. Hum Reprod Update. 2014;20(1):40-62. doi:10.1093/humupd/dmt046

25. Kibat J, Schirrmann T, Knape MJ, et al. Utilisation of antibody microarrays for the selection of specific and informative antibodies from recombinant library binders of unknown quality. $N$ Biotechnol. 2016;33(5PtA):574-581. doi:10.1016/j.nbt.2015.12.003

26. Trayhurn P. Hypoxia and adipose tissue function and dysfunction in obesity. Physiol Rev. 2013;93(1):1-21. doi:10.1152/ physrev.00017.2012

27. Divella R, De Luca R, Abbate I, Naglieri E, Daniele A. Obesity and cancer: the role of adipose tissue and adipo-cytokines-induced chronic inflammation. $J$ Cancer. 2016;7(15):2346-2359. doi:10.7150/jca. 16884

28. Kwaifa IK, Bahari H, Yong YK, Noor SM. Endothelial dysfunction in obesity-induced inflammation: molecular mechanisms and clinical implications. Biomolecules. 2020;10(2):291. doi:10.3390/ biom10020291 
29. Han RY, Ma J, Ma JY, et al. Correlation of semen parameters with inflammatory factors in the seminal plasma of obese males. Zhonghua Nan Ke Xue. 2017;23(10):894-898.

30. Fan W, Xu Y, Liu Y, et al. Obesity or overweight, a chronic inflammatory status in male reproductive system, leads to mice and human subfertility. Front Physiol. 2017;8:1117. doi:10.3389/ fphys.2017.01117

31. Rato L, Alves MG, Cavaco JE, Oliveira PF. High-energy diets: a threat for male fertility? Obes Rev. 2014;15(12):996-1007. doi:10.1111/obr.12226

32. Garolla A, Torino M, Sartini B, et al. Seminal and molecular evidence that sauna exposure affects human spermatogenesis. Hum Reprod. 2013;28(4):877-885. doi:10.1093/humrep/det020
33. Mu Y, Yan WJ, Yin TL, Yang J. Curcumin ameliorates high-fat diet-induced spermatogenesis dysfunction. Mol Med Rep. 2016;14 (4):3588-3594. doi:10.3892/mmr.2016.5712

34. Paasch U, Grunewald S, Agarwal A, et al. Activation pattern of caspases in human spermatozoa. Fertil Steril. 2004;81(suppS1):802-809. doi:10.1016/j.fertnstert.2003.09.030

35. Nagata S. Apoptosis mediated by the fas system. Prog Mol Subcell Biol. 1996;16(16):87.

36. Vergoni B, Cornejo PJ, Gilleron J, et al. DNA damage and the activation of the p53 pathway mediate alterations in metabolic and secretory functions of adipocytes. Diabetes. 2016;65(10):3062-3074. doi:10.2337/db16-0014

Diabetes, Metabolic Syndrome and Obesity: Targets and Therapy

\section{Publish your work in this journal}

Diabetes, Metabolic Syndrome and Obesity: Targets and Therapy is an international, peer-reviewed open-access journal committed to the rapid publication of the latest laboratory and clinical findings in the fields of diabetes, metabolic syndrome and obesity research. Original research, review, case reports, hypothesis formation, expert opinion and commentaries are all considered for publication. The manuscript management system is completely online and includes a very quick and fair peer-review system, which is all easy to use. Visit http://www.dovepress.com/testimonials.php to read real quotes from published authors. 